\title{
A genome-wide association study in Europeans and South Asians identifies five new loci for coronary artery disease
}

\author{
The Coronary Artery Disease (C4D) Genetics Consortium*
}

Genome-wide association studies have identified 11 common variants convincingly associated with coronary artery disease (CAD) ${ }^{1-7}$, a modest number considering the apparent heritability of $\mathrm{CAD}^{8}$. All of these variants have been discovered in European populations. We report a meta-analysis of four large genome-wide association studies of CAD, with $\sim 575,000$ genotyped SNPs in a discovery dataset comprising 15,420 individuals with CAD (cases) (8,424 Europeans and 6,996 South Asians) and 15,062 controls. There was little evidence for ancestry-specific associations, supporting the use of combined analyses. Replication in an independent sample of 21,408 cases and 19,185 controls identified five loci newly associated with CAD $\left(P<5 \times 10^{-8}\right.$ in the combined discovery and replication analysis): LIPA on 10q23, PDGFD on 11q22, ADAMTS7-MORF4L1 on 15q25, a gene rich locus on $7 q 22$ and KIAA1462 on 10p11. The CAD-associated SNP in the PDGFD locus showed tissue-specific cis expression quantitative trait locus effects. These findings implicate new pathways for CAD susceptibility.

Genome-wide association studies (GWAS) in CAD have detected common variants with odds ratios of 1.1-1.3 which in aggregate explain only a small proportion of the predicted genetic risk. We hypothesized that the discovery of new susceptibility loci of smaller effect sizes (and, hence, identification of new CAD-related pathways) would be aided by conducting much larger studies in addition to an emphasis on early onset CAD and clearly defined clinical endpoints.

For the discovery stage, 8,424 cases of European ancestry were recruited by the Precocious Coronary Artery Disease (PROCARDIS) study and Heart Protection Study (HPS), and 6,996 cases of South Asian ancestry (chiefly from Pakistan and India) were recruited by the Pakistan Risk of Myocardial Infarction Study (PROMIS) and London Life Sciences Prospective Population (LOLIPOP) study. All studies recruited controls, or supplemented their data with genotypes from common controls, from within the same self-reported ethnic or linguistic groups from which cases were recruited (Fig. 1). Overall, $81 \%$ of the cases had a prior history of myocardial infarction and the remainder had confirmed diagnoses of symptomatic CAD (angina or coronary artery revascularization), with an average age at first event under 60 years (Supplementary Table 1).

All individuals were typed using whole-genome Illumina BeadChips, allowing for a meta-analysis of actual genotypes rather than imputed data. This enabled analysis of low frequency variants (1-5\%), which have typically been excluded from GWAS either due to sample size or because imputation has been required to combine data from different genotyping platforms.

As there is population substructure within India and Pakistan ${ }^{9}$, principal component analysis ${ }^{10}$ was used in the PROMIS and LOLIPOP studies to identify ancestry informative principal components (Online Methods and Supplementary Fig. 1), which were then used to adjust for population substructure in regression analyses.

Genotypes were tested for association with CAD in the four discovery studies (Online Methods and Supplementary Table 2). Association tests for 574,919 SNPs were entered into pre-specified fixed-effects metaanalyses with study-level correction for genomic control; the meta-analysis groups were: (i) all four studies combined; (ii) the two European studies; and (iii) the two South Asian studies. The genomic control parameters

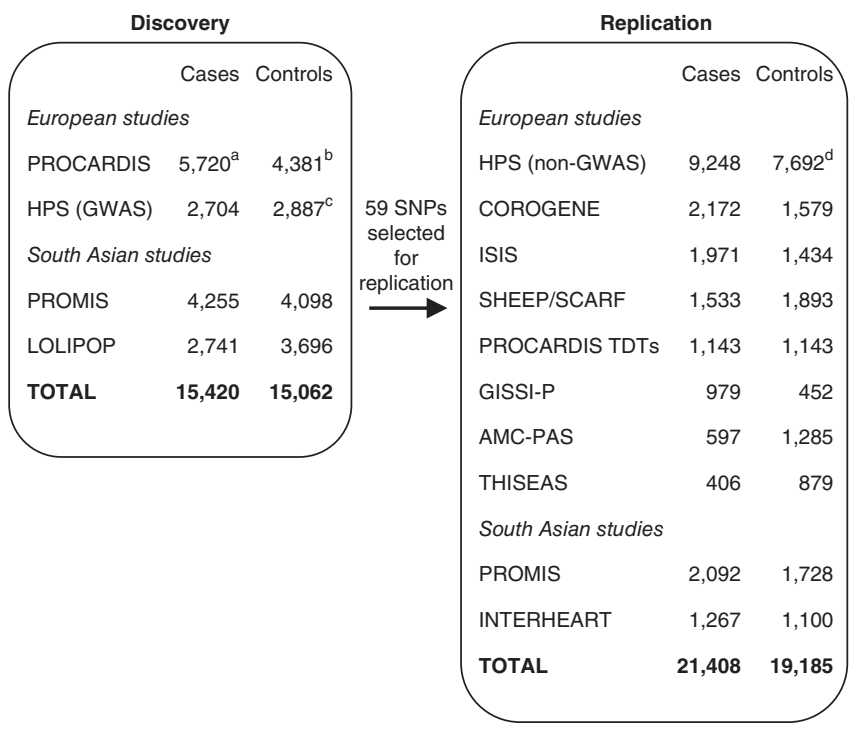

Figure 1 Studies contributing to the discovery and replication metaanalyses. ancludes 2,133 cases who are either full or half siblings of another case. ${ }^{b}$ Includes 2,697 controls from the National Blood Service. 'Includes 2,887 controls from the 1958 British Birth cohort. dincludes 5,157 controls from the UK Twins study and 2,535 additional independent PROCARDIS controls not used in the discovery analysis.

*A full list of authors and affiliations appears at the end of the paper. 


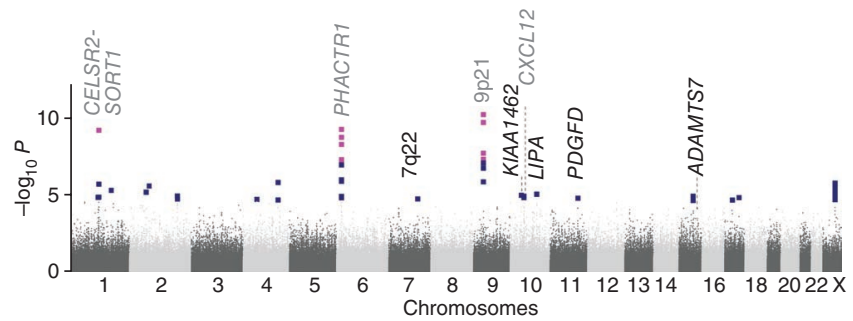

Figure 2 Genome-wide Manhattan plot of $P$ values for all studies (European and South Asian). The $-\log _{10} P$ for 574,919 SNPs from the meta-analysis of the PROCARDIS, HPS, PROMIS and LOLIPOP studies. The $y$ axis is truncated at $-\log _{10} P$ of 12 ; rs9349379 at the PHACTR 1 locus $\left(P=5.8 \times 10^{-19}\right)$ and 15 SNPs at the 9 p21 locus $\left(7.9 \times 10^{-13}>\right.$ $P>1.3 \times 10^{-25}$ ) exceed the truncation. SNP associations with CAD that exceeded the genome-wide significance threshold $\left(P<5.0 \times 10^{-8}\right)$ are shown in magenta; $P$ values between $P=4.5 \times 10^{-5}$ and $P=5.0 \times 10^{-8}$ are shown in blue. The locations of the new replicated loci are annotated in black and previously reported CAD loci (Table 1 ) with $P<4.0 \times 10^{-5}$ in the meta-analysis of all studies together are annotated in gray.

$\left(\lambda_{\mathrm{GC}}\right)$ after the study-level genomic control correction were 1.03 for the four studies combined, 1.01 for the European studies only and 1.01 for the South Asian studies only; a further meta-analysis-level genomic control correction was applied to each meta-analysis. Heterogeneity was tested between the four discovery GWAS and between the European and South Asian meta-analyses. Manhattan plots are shown in Figure 2 for the meta-analysis of all studies combined and in Supplementary Figure 2 for the ancestry-specific meta-analyses, and the quantile-quantile plots are shown in Supplementary Figure 3.

We confirmed the power and representative nature of our discovery-stage studies with data supporting the relevance of 11 known CAD susceptibility loci with comparable effect sizes to those reported previously (Table 1). We saw directionally consistent effects in the European and South Asian populations for all 11 loci.

We selected 59 SNPs from 50 loci that showed potential new associations from the meta-analysis of the European and South Asian studies (41 SNPs; $P<1.0 \times 10^{-4}$ ), the European only meta-analysis (8 SNPs; $P<3.0 \times 10^{-5}$ ), the South Asian only meta-analysis (6 SNPS; $\left.P<3.0 \times 10^{-5}\right)$ and three loci with strong biological plausibility but only suggestive $P$ values (4 SNPs). These SNPs were tested in ten replication studies involving a total of 21,408 CAD cases and 19,185 controls largely by de novo genotyping (Fig. 1). The meta-analysis of the replication association results is shown in Supplementary Table 3. Five SNPs in the newly associated loci achieved the prespecified threshold for replication $\left(P<8.5 \times 10^{-4}\right.$; which is $P<0.05$ after Bonferroni correction for 59 independent tests), and each also achieved conventional genome-wide significance $\left(P<5.0 \times 10^{-8}\right)$, with $P$ values ranging from $2.8 \times 10^{-13}$ to $3.9 \times 10^{-8}$ for the combined discovery and replication meta-analysis (Fig. 3). Apparent heterogeneity between the European and South Asian effect for rs4380028 in the ADAMTS7-MORF4L1 locus in the discovery meta-analysis was not supported by the independent replication. We observed no evidence of ancestry-specific heterogeneity for any of the other previously unidentified loci in either the discovery or replication meta-analyses.

In addition to the five newly associated loci, rs9349379, located in an intron of PHACTR1, was significantly associated in the replication alone $\left(P=9.9 \times 10^{-10}\right)$ and in the combined discovery and replication $\left(P=8.7 \times 10^{-26}\right)$ meta-analyses, and rs17114046, in an intron of $P P A P 2 B$, showed consistent support in the discovery and replication meta-analyses but fell outside the pre-determined significance level in both the replication alone $\left(P=1.1 \times 10^{-3}\right)$ and in the combined discovery and replication $\left(P=2.5 \times 10^{-7}\right)$ metaanalyses. It is plausible that other SNPs in the replication study that had suggestive associations have a real effect on CAD; a quantilequantile plot (Supplementary Fig. 3) of the replication study results shows an overdispersion of the test statistic.

The regional association plots for each confirmed locus are shown in Figure 4. We performed analyses conditioning on the replicated SNP at each new locus in all four discovery studies, and the meta-analysis of these results revealed no evidence of additional independent associations with CAD.

We investigated associations between the expression levels of all genes within $200 \mathrm{~kb}$ of each of the confirmed risk SNPs in tissue samples of aortic media and adventitia, mammary artery, carotid plaque, liver, adipose tissue, transformed lymphoblastoid cell lines and skin. The Bonferroni-adjusted significance threshold was $P<$ $3.1 \times 10^{-4}(P<0.05$ over 163 tests $)$. Estimates of genotype effect on gene expression are reported in Supplementary Table 4 for the most significantly associated gene in each search window and, when different from the risk SNP, the most significant expression quantitative trait locus (eQTL) SNP.

None of these loci has any previously reported associations with established CAD risk factors (lipids, blood pressure, glucometabolic traits or body mass index). rs4380028 in the ADAMTS7-MORF4L1

Table 1 Evidence in the discovery studies for 11 previously reported common variants associated with CAD in GWAS

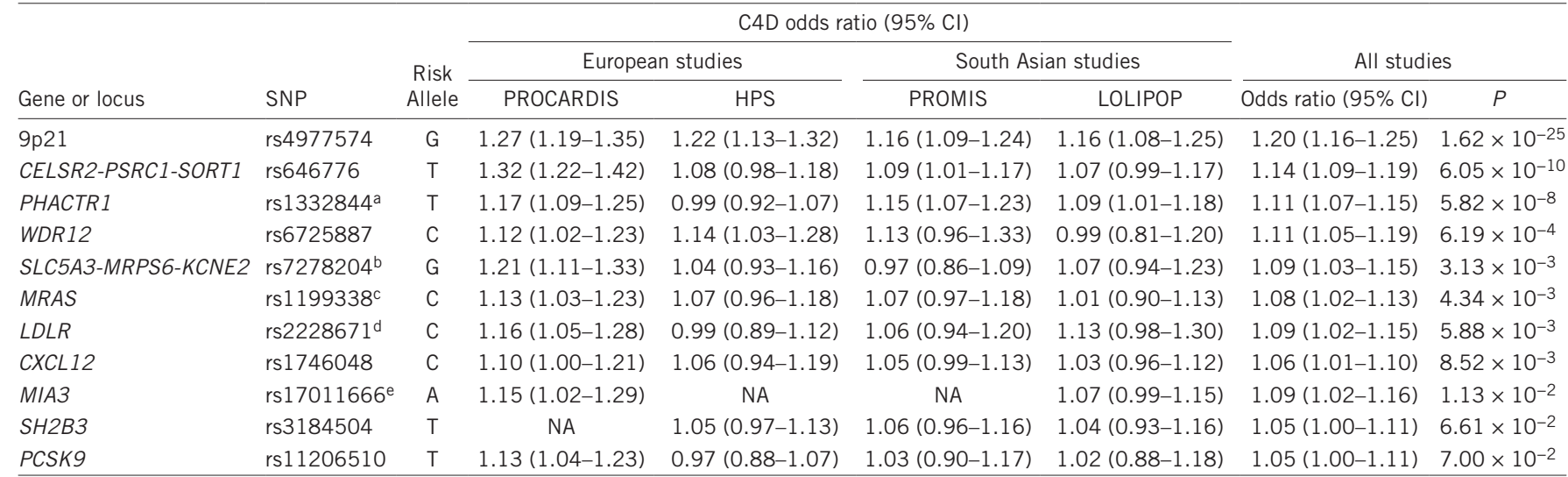

Odds ratios per risk allele and $95 \% \mathrm{Cls}$ for 11 SNPs previously associated with $C A D^{1,3-7,19}$ are reported in the discovery studies separately and overall.

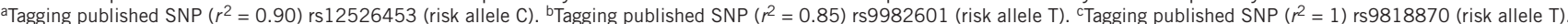

dTagging published SNP $\left(r^{2}=0.73\right)$ rs1122608 (risk allele G). ${ }^{2}$ Tagging published SNP $\left(r^{2}=0.57\right)$ rs17465637 (risk allele C). NA, not available. 
The nearest gene to rs974819 is PDGFD, $117 \mathrm{~kb}$ downstream in an adjacent block of linkage disequilibrium (LD) (Fig. 4). We found a significant $P D G F D$ eQTL for rs974819 in aortic media $(P<2.3 \times$ $\left.10^{-7}\right)$, with suggestive associations in aortic adventitia $\left(P<7.7 \times 10^{-4}\right)$ and mammary artery $\left(P<7.2 \times 10^{-4}\right)$ (Supplementary Table 4$)$. In all three tissues, the risk allele was associated with increased expression, and PDGFD was one of the top $10 \%$ most highly expressed transcripts. Platelet-derived growth factor $\mathrm{D}$, encoded by $P D G F D$, is expressed in several cell types in atherosclerotic plaques and is predicted to stimulate atherosclerosis by influencing matrix metalloproteinase activity and monocyte migration ${ }^{12}$ and by inhibiting smooth muscle cell gene expression ${ }^{13}$.

rs4380028 is $7.6 \mathrm{~kb}$ upstream of ADAMTS7. ADAMTS7 is a metalloproteinase that accumulates in carotid artery neointima after injury and is upregulated by platelet-derived growth factor ${ }^{14}$. The CAD association at the ADAMTS7 locus appears to be independent of the adjacent QTL for cigarette smoking, which has been attributed to the CHRNA5-CHRNA3-CHRNB4 nicotinic acid receptor cluster ${ }^{15}$. The signal at rs 4380028 therefore appears new but cannot be attributed to a specific gene.

At the 7q22 locus, the lead SNP, rs10953541, is within an intron of $B C A P 29$, which encodes $\mathrm{B}$-cell receptor-associated protein 29 , but this SNP is also in strong LD with five other protein coding genes (PRKAR2B, HBP1, COG5, GPR22 and DUS4L). We observed no unique eQTL effect. One promising biological candidate is GPR22, which encodes a G-protein-coupled receptor expressed in coronary arteries and heart ${ }^{16}$.

rs2505083 is in an intron of KIAA1462, a widely expressed and evolutionarily conserved gene. The function of the 1,359-amino-acid protein encoded by KIAA1462 is largely unknown, with no recognizable functional domains and little homology to other protein families.

The genetic evidence for rs17114046 in PPAP2B is further supported by the association of the risk allele, with a suggestive $(P<3.9$ $\left.\times 10^{-3}\right) 30 \%$ increase in $P P A P 2 B$ expression in atherosclerotic plaque (Supplementary Table 4). $P P A P 2 B$ encodes phosphatidic acid phosphatase type $2 \mathrm{~B}$, a membrane glycoprotein that hydrolyzes bioactive lipids involved in signaling ${ }^{17}$. It is expressed in the adherens junctions of endothelial cells and is believed to influence endothelial cell adhesion and migration and vasculogenesis ${ }^{18}$.

In summary, five new loci passed both the pre-specified significance threshold for the stand-alone replication and the conventional threshold for genome-wide significance in the combined discovery and replication data, yielding a substantial increase in the number of confirmed susceptibility loci for CAD. We did not find any susceptibility variants with material differences in effect size or allele frequency between South Asians and Europeans. We note, however, the potential limitation that current genome-wide arrays may not capture all important variants in South Asians. Nevertheless, all of the known and new variants were significantly associated with CAD risk in both the European and South Asian populations in the current study, indicating the importance of the genes associated with CAD beyond the European ancestry groups in which they were first defined.

As is seen in the present study, the effect sizes of previously unidentified CAD-associated genes discovered by GWAS have become progressively smaller, suggesting that there may not be large-effect common variants remaining to be discovered, but rather that a large number of common variants of small effect may contribute to CAD risk. Reliable detection of differences of only $5-10 \%$ in the per-allele risk of CAD has previously been difficult. However, the availability of large-scale GWAS carried out in populations of different ancestry, and our demonstration that results from such populations can be informatively combined in genetic discovery, suggests that even broader collaborations would identify additional variants that influence CAD risk. Greater understanding of the genetic variants underlying $\mathrm{CAD}$, and particularly the pathways involved, may lead to development of new therapeutic approaches to help address the world's leading cause of death.

URLs. TRANSMIT software, http://www-gene.cimr.cam.ac.uk/ clayton/software/transmit.txt; METAL software, http://www.sph. umich.edu/csg/abecasis/Metal/.

Note added in proof: Since this manuscript was submitted ADAMTS7 (ref. 20) and KIAA1462 (ref. 21) have been independently reported as loci for coronary artery disease.

\section{METHODS}

Methods and any associated references are available in the online version of the paper at http://www.nature.com/naturegenetics/.

Note: Supplementary information is available on the Nature Genetics website.

\section{ACKNOWLEDGMENTS}

We are grateful to all of the study participants in the studies contributing to these meta-analyses and to all laboratory staff and former colleagues who have contributed to these studies over many years. This study makes use of data generated by the Wellcome Trust Case-Control Consortium (WTCCC); a full list of the investigators who contributed to the generation of the data is available from www.wtccc.org.uk. This study also makes use of data generated by the UK Twins study, The Twin Research Unit, King's College London, UK.

The PROCARDIS study was supported by the European Community Sixth Framework Program (LSHM-CT-2007-037273), AstraZeneca, the British Heart Foundation, the Oxford British Heart Foundation Centre of Research Excellence, the Wellcome Trust (075491/Z/04), the Swedish Research Council, the Knut and Alice Wallenberg Foundation, the Swedish Heart-Lung Foundation, the Torsten and Ragnar Söderberg Foundation, the Strategic Cardiovascular Program of Karolinska Institutet and Stockholm County Council, the Foundation for Strategic Research and the Stockholm County Council (560283).

The Heart Protection Study (ISRCTN48489393) was funded by the UK Medical Research Council, the British Heart Foundation, Merck \& Co and Roche Vitamins Ltd. Genotyping and analysis was supported by a grant to Oxford University and the Centre National de Génotypage (CNG) from Merck \& Co and the Oxford BHF Centre of Research Excellence.

The PROMIS study was funded by unrestricted grants to investigators at the University of Cambridge, UK and at the Centre for Non-Communicable Diseases, Pakistan. Genotyping was funded by the Wellcome Trust.

The LOLIPOP study is supported by the National Institute for Health Research Comprehensive Biomedical Research Centre Imperial College Healthcare NHS Trust, Ealing Hospital NHS Trust, the British Heart Foundation (SP/04/002), the Medical Research Council (G0700931, G0601966), the Wellcome Trust (084723/Z/ $08 / \mathrm{Z}$ ) and the National Institute for Health Research (RP-PG-0407-10371). P.E. is a National Institute for Health Research Senior Investigator. This work was facilitated by Barts and The London National Institute for Health Biomedical Research Unit. We thank the participants and research staff who made the study possible.

The COROGENE-FINRISK study was supported in part by the Aarno Koskelo Foundation and the Finnish Foundation for Cardiovascular Research.

The Biobank of Karolinska Carotid Endarterectomies (BiKE) and Advanced Study of Aortic Pathology (ASAP) eQTL study was supported by the Swedish HeartLung Foundation, the Swedish Research Council, the European Commission (FAD, Health-F22008-200647), (AtheroRemo HEALTH-2007-A-201668), DASTI (Danish Agency for Science, Technology and Innovation) and a donation from F. Lundberg.

The Multiple Tissue Human Expression Resource (MuTHER) study was supported by the Wellcome Trust $(081917 / \mathrm{Z} / 07 / \mathrm{Z})$.

N. Soranzo is supported by the Wellcome Trust (Core Grant Number 091746/Z/10/Z).

\section{AUTHOR CONTRIBUTIONS}

Steering and writing committee: J.F.P., J.C.H., D.S., J.C.C., J.H., N. Soranzo, R. Collins, J.D., P. Elliott, M.F., K.S., W.Z., A. Hamsten, S. Parish, M.L., H.W. (Chair), R. Clarke, P. Deloukas, J.S.K.

Corresponding authors: H.W., D.S., R. Collins, J.S.K.

Analysis committee: J.C.H., W.Z., N. Soranzo, J.F.P., D.S., J.C.C., S. Parish, M.F. (Chair). 
Statistical genetics and bioinformatics: HPS: J.C.H., S. Parish; LOLIPOP: W.Z.; PROCARDIS: A. Goel, H.O., R.J.S., S.H., A.M., A. Helgadottir, J.O., M.F., J.F.P.; PROMIS: D.S., K.S., M.M., S. Potter, S.E.H., P. Deloukas.

Genotyping: CNG: J.H., M.D., M.L.; Karolinska: R.J.S.; Oxford: S.J., H.O.; Uppsala: T.A., A.C.S.; WTSI: R.G., S. Bumpsted, E.G., S.E., P.D.

Expression QTL analyses: L.F., T.K., A.F.C., A. Gabrielsen, U.S., the MuTHER consortium, P. Eriksson.

Discovery cohorts: HPS: J.C.H., S. Parish, A.O., R. Clarke, L.B., P.S., J.A., R.P., R. Collins; LOLIPOP: J.C.C., G. Abecasis, N.A., M.C., P. Donnelly, P. Elliott, P.F., A.S.K., M.I.C., N.J.S., J. Scott, J. Sehmi, W.Z., J.S.K.; PROCARDIS: Sweden: A. Silveira, M.L.H., F.M.v.H., G.O., A. Hamsten; Germany: S. Rust, G. Assmann, U.S.; Italy: S. Barlera, G.T., M.G.F.; UK: R. Clarke, P.L., J.C.H., R. Collins, J.F.P., F.R.G., M.F., H.W.; PROMIS: D.S., A.R., M.Z., N. Shah, M.S., N.H.M., M.A., K.S.Z., A. Samad, M. Ishaq, A.R.G., F.M., N.J.S., P.M.F., P.D., J.D.

Replication cohorts: UK Twins: N. Soranzo, T.S.; COROGENE-FINRISK: L.P., M.S.N., J. Sinisalo, V.S., S. Ripatti; ISIS: J.C.H., D.B., S. Parish; SHEEP/SCARF: K.L., B.G., U.d.F.; GISSI-P: S. Pietri, F.G., R.M.; AMC-PAS: S.S., J.J.P.K., M.D.T.; THISEAS: E.V.T., G.V.D.; INTERHEART: J.C.E., S.Y., S.S.A.

For further details on author contributions, see the Supplementary Note.

\section{COMPETING FINANCIAL INTERESTS}

The authors declare no competing financial interests.

\section{Published online at http://www.nature.com/naturegenetics/.}

Reprints and permissions information is available online at http://npg.nature.com/ reprintsandpermissions/.

1. Erdmann, J. et al. New susceptibility locus for coronary artery disease on chromosome 3q22.3. Nat. Genet. 41, 280-282 (2009).

2. Helgadottir, A. et al. A common variant on chromosome $9 p 21$ affects the risk of myocardial infarction. Science 316, 1491-1493 (2007).

3. Kathiresan, S. et al. Genome-wide association of early-onset myocardial infarction with single nucleotide polymorphisms and copy number variants. Nat. Genet. 41, 334-341 (2009).

4. McPherson, R. et al. A common allele on chromosome 9 associated with coronary heart disease. Science 316, 1488-1491 (2007).

5. Samani, N.J. et al. Genome-wide association analysis of coronary artery disease. N. Engl. J. Med. 357, 443-453 (2007).
6. Soranzo, N. et al. A genome-wide meta-analysis identifies 22 loci associated with eight hematological parameters in the HaemGen consortium. Nat. Genet. 41 , 1182-1190 (2009).

7. Wellcome Trust Case Control Consortium. Genome-wide association study of 14,000 cases of seven common diseases and 3,000 shared controls. Nature 447, 661-678 (2007).

8. Zdravkovic, S. et al. Heritability of death from coronary heart disease: a 36-year follow-up of 20966 Swedish twins. J. Intern. Med. 252, 247-254 (2002).

9. Reich, D., Thangaraj, K., Patterson, N., Price, A.L. \& Singh, L. Reconstructing Indian population history. Nature 461, 489-494 (2009).

10. Price, A.L. et al. Principal components analysis corrects for stratification in genomewide association studies. Nat. Genet. 38, 904-909 (2006).

11. Zeller, T. et al. Genetics and beyond-the transcriptome of human monocytes and disease susceptibility. PLOS ONE 5, e10693 (2010).

12. Wagsater, D., Zhu, C., Bjorck, H.M. \& Eriksson, P. Effects of PDGF-C and PDGF-D on monocyte migration and MMP-2 and MMP-9 expression. Atherosclerosis 202, 415-423 (2009).

13. Thomas, J.A. et al. PDGF-DD, a novel mediator of smooth muscle cell phenotypic modulation, is upregulated in endothelial cells exposed to atherosclerosis-prone flow patterns. Am. J. Physiol. Heart Circ. Physiol. 296, H442-H452 (2009).

14. Wang, L. et al. ADAMTS-7 mediates vascular smooth muscle cell migration and neointima formation in balloon-injured rat arteries. Circ. Res. 104, 688-698 (2009).

15. Tobacco and Genetics Consortium. Genome-wide meta-analyses identify multiple loci associated with smoking behavior. Nat. Genet. 42, 441-447 (2010).

16. Adams, J.W. et al. Myocardial expression, signaling, and function of GPR22: a protective role for an orphan G protein-coupled receptor. Am. J. Physiol. Heart Circ. Physiol. 295, H509-H521 (2008)

17. Brindley, D.N. \& Pilquil, C. Lipid phosphate phosphatases and signaling. J. Lipid Res. 50 Suppl, S225-S230 (2009).

18. Humtsoe, J.O., Liu, M., Malik, A.B. \& Wary, K.K. Lipid phosphate phosphatase 3 stabilization of beta-catenin induces endothelial cell migration and formation of branching point structures. Mol. Cell. Biol. 30, 1593-1606 (2010).

19. Schunkert, H. et al. Repeated replication and a prospective meta-analysis of the association between chromosome 9p21.3 and coronary artery disease. Circulation 117, 1675-1684 (2008).

20. Reilly, M.P. et al. Identification of ADAMTS7 as a novel locus for coronary atherosclerosis and association of $\mathrm{ABO}$ with myocardial infarction in the presence of coronary atherosclerosis: two genome-wide association studies. Lancet 377, 383-292 (2011)

21. Erdmann, J. et al. Genome-wide association study identifies a new locus for coronary artery disease on chromosome 10p11.23. Eur. Heart J. 32, 158-168 (2011).

Steering and Writing committee: John F Peden ${ }^{1,2,54}$, Jemma C Hopewell ${ }^{3,54}$, Danish Saleheen ${ }^{4,5,54}$, John C Chambers ${ }^{6,7,54}$, Jorg Hager ${ }^{8,54}$, Nicole Soranzo ${ }^{9,54}$, Rory Collins ${ }^{3,54}$, John Danesh ${ }^{5,54}$, Paul Elliott ${ }^{6,10,54}$, Martin Farrall ${ }^{1,2,54}$, Kathy Stirrups ${ }^{9,54}$, Weihua Zhang ${ }^{6,7,54}$, Anders Hamsten ${ }^{11,12,54}$, Sarah Parish ${ }^{3,54}$, Mark Lathrop ${ }^{8,54}$, Hugh Watkins (Chair) ${ }^{1,2,54}$, Robert Clarke ${ }^{3,54}$, Panos Deloukas ${ }^{9,54}$ \& Jaspal S Kooner ${ }^{7,13,54}$

Statistical genetics and bioinformatics: Anuj Goel ${ }^{1,2}$, Halit Ongen ${ }^{1,2}$, Rona J Strawbridge ${ }^{11,12}$, Simon Heath ${ }^{8}$, Anders Mälarstig ${ }^{11,12}$, Anna Helgadottir ${ }^{1,2}$, John Öhrvik ${ }^{11,12}$, Muhammed Murtaza , Simon Potter ${ }^{14} \&$ Sarah E Hunt ${ }^{14}$

Genotyping: Marc Delepine ${ }^{8}$, Shapour Jalilzadeh ${ }^{1,2}$, Tomas Axelsson ${ }^{15}$, Ann-Christine Syvanen ${ }^{15}$, Rhian Gwilliam ${ }^{14}$, Suzannah Bumpstead ${ }^{14}$, Emma Gray ${ }^{14}$ \& Sarah Edkins ${ }^{14}$

Expression QTL analyses: Lasse Folkersen ${ }^{11,12}$, Theodosios Kyriakou ${ }^{1,2}$, Anders Franco-Cereceda ${ }^{16}$, Anders Gabrielsen ${ }^{17}$, Udo Seedorf ${ }^{18}$, the MuTHER consortium \& Per Eriksson ${ }^{11,12}$

Discovery cohorts: Alison Offer ${ }^{3}$, Louise Bowman ${ }^{3}$, Peter Sleight ${ }^{2}$, Jane Armitage ${ }^{3}$, Richard Peto ${ }^{3}$, Goncalo Abecasis $^{19}$, Nabeel Ahmed ${ }^{20}$, Mark Caulfield ${ }^{21}$, Peter Donnelly ${ }^{22,23}$, Philippe Froguel ${ }^{24}$, Angad S Kooner ${ }^{20}$, Mark I McCarthy ${ }^{22,25,26}$, Nilesh J Samani ${ }^{27,28}$, James Scott ${ }^{13}$, Joban Sehmi ${ }^{13}$, Angela Silveira ${ }^{11,12}$, Mai-Lis Hellénius ${ }^{29}$, Ferdinand $M$ van 't Hooft ${ }^{11,12}$, Gunnar Olsson ${ }^{30,31}$, Stephan Rust ${ }^{18}$, Gerd Assmann $^{18}$, Simona Barlera ${ }^{32}$, Gianni Tognoni ${ }^{32,33}$, Maria Grazia Franzosi ${ }^{32}$, Pamela Linksted ${ }^{3}$, Fiona R Green ${ }^{34}$, Asif Rasheed ${ }^{4}$, Moazzam Zaidi ${ }^{4}$, Nabi Shah ${ }^{4}$, Maria Samuel ${ }^{4}$, Nadeem H Mallick ${ }^{35}$, Muhammad Azhar ${ }^{35}$, Khan S Zaman ${ }^{36}$, Abdus Samad ${ }^{37}$, Mohammad Ishaq ${ }^{37}$, Ali R Gardezi ${ }^{38}$, Fazal-ur-Rehman Memon ${ }^{39}$ \& Philippe M Frossard ${ }^{4}$

Replication cohorts: Tim Spector ${ }^{40}$, Leena Peltonen ${ }^{9,41,45}$, Markku S Nieminen ${ }^{42}$, Juha Sinisalo ${ }^{43}$, Veikko Salomaa $^{44}$, Samuli Ripatti ${ }^{44,45}$, Derrick Bennett ${ }^{3}$, Karin Leander ${ }^{46}$, Bruna Gigante ${ }^{46}$, Ulf de Faire ${ }^{46}$, 


\title{
Silvia Pietri ${ }^{32}$, Francesca Gori ${ }^{32}$, Roberto Marchioli ${ }^{47}$, Suthesh Sivapalaratnam ${ }^{48}$, John J P Kastelein ${ }^{48}$, Mieke D Trip ${ }^{48}$, Eirini V Theodoraki ${ }^{49}$, George V Dedoussis ${ }^{49}$, Jamie C Engert ${ }^{50,51}$, Salim Yusuf ${ }^{52}$ \& Sonia S Anand ${ }^{52,53}$
}

\begin{abstract}
${ }^{1}$ Department of Cardiovascular Medicine, The Wellcome Trust Centre for Human Genetics, University of Oxford, Oxford, UK. 2Department of Cardiovascular Medicine, University of Oxford, John Radcliffe Hospital, Headington, Oxford, UK. ${ }^{3}$ Clinical Trial Service Unit, University of Oxford, Oxford, UK. ${ }^{4}$ Center for Non-Communicable Diseases Pakistan, Karachi, Pakistan. ${ }^{5}$ Department of Public Health and Primary Care, University of Cambridge, Strangeways Research Laboratory, Cambridge, UK. ${ }^{6}$ Epidemiology and Biostatistics, Imperial College London, Norfolk Place, London, UK. ${ }^{7}$ Cardiology, Ealing Hospital National Health Service (NHS) Trust, Middlesex, UK. ${ }^{8}$ Commissariat à I'Energie Atomique (CEA) Genomics Institute-Centre National de Génotypage, Evry Cedex, France. ${ }^{9}$ Human Genetics, Wellcome Trust Sanger Institute, Wellcome Trust Genome Campus, Hinxton, UK. ${ }^{10}$ Medical Research Council-Health Protection Agency (MRC-HPA) Centre for Environment and Health, Imperial College London, London, UK. ${ }^{11}$ Atherosclerosis Research Unit, Department of Medicine, Karolinska Institutet, Karolinska University Hospital, Stockholm, Sweden. ${ }^{12}$ Center for Molecular Medicine, Karolinska University Hospital, Stockholm, Sweden. ${ }^{13}$ National Heart and Lung Institute, Imperial College London, London, UK. ${ }^{14}$ Wellcome Trust Sanger Institute, Wellcome Trust Genome Campus, Hinxton, UK. ${ }^{15}$ Department of Medical Sciences, Molecular Medicine, Uppsala University, Uppsala, Sweden. ${ }^{16}$ Thoracic Surgery Unit, Department of Molecular Medicine and Surgery, Karolinska Institutet, Karolinska University Hospital, Stockholm, Sweden. ${ }^{17}$ Cardiology Unit, Department of Medicine, Karolinska Institutet, Karolinska University Hospital, Stockholm, Sweden. ${ }^{18}$ Gesellschaft für Arterioskleroseforschung e.V., Leibniz-Institut für Arterioskleroseforschung an der Universität Münster (LIFA), Münster, Germany. ${ }^{19}$ Department of Biostatistics, University of Michigan, Ann Arbor, Michigan, USA. ${ }^{20}$ Cardiology, Ealing Hospital NHS Trust, Middlesex, UK. ${ }^{21}$ William Harvey Research Institute, Barts and The London School of Medicine and Dentistry, Queen Mary University of London, London, UK. ${ }^{22}$ Wellcome Trust Centre for Human Genetics, University of Oxford, Oxford, UK. ${ }^{23}$ Department of Statistics, Oxford University, Oxford, UK. ${ }^{24}$ Genomic Medicine, Imperial College London, London, UK. ${ }^{25}$ Oxford Centre for Diabetes, Endocrinology and Metabolism, Oxford University, Oxford, UK. ${ }^{26}$ Oxford National Institute of Health Research (NIHR) Biomedical Research Centre, Churchill Hospital, Headington, UK. ${ }^{27}$ Department of Cardiovascular Sciences, University of Leicester, Glenfield Hospital, Leicester, UK. ${ }^{28}$ Leicester NIHR Biomedical Research Unit in Cardiovascular Disease, Glenfield Hospital, Leicester, UK. ${ }^{29}$ Cardiology Unit, Department of Medicine, Karolinska Institutet, Karolinska University Hospital, Stockholm, Sweden. ${ }^{30}$ Cardiovascular Drug Research at the Department of Medicine, Solna, Sweden. ${ }^{31}$ Cardiovascular and Gastrointestinal Innovative Medicines, Global Research and Development, AstraZeneca, Sweden. ${ }^{32}$ Department of Cardiovascular Research, Istituto Mario Negri, Milano, Italy. ${ }^{33}$ Consorzio Mario Negri Sud, Santa Maria Imbaro (Chieti), Italy. ${ }^{34}$ Biochemical Sciences Division, Faculty of Health and Medical Sciences, University of Surrey, Guilford, UK. ${ }^{35}$ Department of Cardiology, Punjab Institute of Cardiology, Jail Road, Lahore, Pakistan. ${ }^{36}$ Department of Cardiology, National Institute of Cardiovascular Diseases, Karachi, Pakistan. ${ }^{37}$ Department of Cardiology, Karachi Institute of Heart Diseases, Federal B. Area, Karachi, Pakistan. ${ }^{38}$ Department of Cardiology, Ch. Pervaiz Elahi Institute Of Cardiology, Multan, Pakistan. ${ }^{39}$ Department of Cardiology, Red Crescent Institute of Cardiology, Latifabad, Hyderabad, Pakistan. ${ }^{40}$ Department of Twin Research and Genetic Epidemiology, Kings College London, London, UK. ${ }^{41}$ Department of Medical Genetics, University of Helsinki and the Helsinki University Central Hospital, Helsinki, Finland. ${ }^{2}$ Division of Cardiology, Helsinki University Central Hospital, Helsinki, Finland. ${ }^{43}$ University Central Hospital, Cardiovascular Laboratory, Helsinki, Finland. ${ }^{44}$ National Institute for Health and Welfare, Helsinki, Finland. ${ }^{45}$ Institute for Molecular Medicine Finland (FIMM), University of Helsinki, Helsinki, Finland. ${ }^{46}$ Cardiovascular Epidemiology Unit, Institute of Environmental Medicine, Karolinska Institutet, Stockholm, Sweden. ${ }^{47}$ Laboratory of Clinical Epidemiology of Cardiovascular Disease, Consorzio Mario Negri Sud, Santa Maria Imbaro, Italy. ${ }^{48}$ Vascular Medicine, Academic Medical Center, University of Amsterdam, Amsterdam, The Netherlands. ${ }^{49}$ Department of Dietetics-Nutrition, Harokopio University, Athens, Greece. ${ }^{50}$ McGill University Department of Medicine, Montreal, Quebec, Canada. ${ }^{51}$ McGill University Department of Human Genetics, Montreal, Quebec, Canada. ${ }^{52}$ Population Health Research Institute, Hamilton Health Sciences, McMaster University, Hamilton, Canada. ${ }^{53}$ Department of Medicine, McMaster University, Hamilton, Canada. ${ }^{54}$ These authors contributed equally to this work. Correspondence should be addressed to H.W. (hugh.watkins@cardiov.ox.ac.uk), D.S. (DS436@medschl.cam.ac.uk), R.C. (rory.collins@ctsu.ox.ac.uk) or J.S.K. (j.kooner@imperial.ac.uk).
\end{abstract}




\section{ONLINE METHODS}

Discovery. Cases and controls in the discovery studies. The PROCARDIS study comprised individuals recruited from the UK, Italy, Sweden and Germany ${ }^{22}$. All cases had a diagnosis of CAD before age 66 and $80 \%$ had a sibling in whom CAD had been diagnosed before age 66 . CAD was defined as clinically documented evidence of myocardial infarction $(80 \%)$, coronary artery bypass graft $(10 \%)$, acute coronary syndrome $(6 \%)$, coronary angioplasty $(1 \%)$ or stable angina (hospitalization for angina or documented obstructive coronary disease) (3\%) before age 66. The mean age of onset of CAD was 53.2 years (standard deviation (s.d.), 7.2 years).

The Heart Protection Study (HPS) was a large UK-based cholesterol-lowering trial involving participants with a history of myocardial infarction, unstable or stable angina, coronary artery bypass graft or coronary angioplasty (as well as individuals with prior history of stroke or hypertension $)^{23}$. Among these, 2,704 CAD cases were genotyped and compared with 2,887 controls from the UK 1958 British Birth Cohort. Of those individuals genotyped, the mean age of CAD onset was 58.8 years (s.d., 8.4 years), and $92 \%$ of CAD cases had a history of myocardial infarction, revascularization or hospitalization for angina.

The PROMIS study was a case-control study of myocardial infarction carried out among South Asians living in urban Pakistan ${ }^{24}$. The 4,253 cases had myocardial infarction, and the average age of disease onset was 53.8 years (s.d., 10.6 years). The 4,130 controls were matched to cases by sex and age and recruited in the same hospitals as the index cases. The major ethnic groups of the participants in the PROMIS study were as follows: Urdu (42\%), Punjabi (28\%), Pathan (8\%) and Sindhi (8\%).

The LOLIPOP study was a case-control study of CAD carried out among South Asians, a population category defined by having all four grandparents born on the Indian subcontinent, living in the $\mathrm{UK}^{25}$. The 2,741 cases had a history of myocardial infarction, coronary artery revascularization (coronary artery bypass grafting or percutaneous coronary intervention) or angiographically confirmed coronary artery stenosis greater than $50 \%$. The cases were compared with 3,696 controls, free from CAD, recruited from the same ethnic or linguistic groups. The mean age of entry into the study was 59.3 years (s.d., 9.7 years). For further details of the discovery cohorts, see the Supplementary Note.

Genotyping in the discovery studies. The genotyping platforms, centers and quality control parameters are summarized in Supplementary Table 2. Additional quality control was done by testing for different allelic frequencies between centers, bead arrays, batches or BeadChips, and a further 3,883 SNPs were removed.

To identify ancestry outliers, principal component analysis using EIGENSOFT (3.0) ${ }^{10}$ was used to compare all samples with reference samples from the HapMap YRI, CHB, JPT and CEU panels. Samples with eigenvalues inconsistent with self-reported ancestry were removed. To detect and correct for population stratification, a panel of independent SNPs were used to generate ancestry-informative principal components. South Asian samples were plotted by their self-reported ethnic or linguistic group and case or control status (Supplementary Fig. 1).

Ethics. All studies were collected under the approval of the appropriate ethics committee and all participants gave informed consent for each study.

Statistical analyses. Discovery genome-wide analyses. Association analyses of CAD were carried out after exclusion of SNPs that failed quality control. Analyses were performed using logistic models to estimate regression coefficients and their standard errors. Additive genetic effects were modeled by defining continuous variables with levels 0,1 and 2 corresponding to genotypes $\mathrm{AA}, \mathrm{AB}$ and $\mathrm{BB}$. The test statistics for each study were inspected for over-dispersion, and the genomic control parameter $\left(\lambda_{\mathrm{GC}}\right)$, an estimate of the variance inflation, was between 1.03 to 1.08 (Supplementary Table 2).

Clustering and covariate adjustment. The multi-center PROCARDIS study included country of origin as a categorical main effect to model differences in SNP allele frequencies indirectly across the populations. The familial relatedness in the PROCARDIS study was taken into account using a robust (sandwich) estimator of the variance. The PROMIS and LOLIPOP studies included ancestry-informative principal components to absorb population stratification.
Discovery meta-analysis. A fixed-effects inverse variance-weighted metaanalysis as implemented by METAL (see URLs) was used to combine the individual studies concurrently as European studies only, South Asian studies only and all studies. The individual studies were subjected to study-level correction for genomic control (using the sample-specific $\lambda_{\mathrm{GC}}$ described above). The test statistics for each meta-analysis were inspected for over-dispersion (Supplementary Fig. 3). The $\lambda_{\mathrm{GC}}$ of the three meta-analyses were calculated ( $\lambda_{\mathrm{GC}}=1.01$ Europeans only, $\lambda_{\mathrm{GC}}=1.01$ South Asians only and $\lambda_{\mathrm{GC}}=1.03$ for all studies), and an additional genomic control correction was applied using these $\underline{\lambda}_{\mathrm{GC}}$ factors (double genomic control correction). Heterogeneity between the European and the South Asian studies and between all studies was assessed using the Cochran's $Q$ statistic.

Replication. Cases and controls in the replication studies. Independent replication was sought in a total of 21,408 CAD cases and 19,185 ancestry-matched controls obtained from eight European and two South Asian studies. A total of 18,049 CAD cases and 16,357 controls of European ancestry were included from HPS $^{23}$, COROGENE-FINRISK ${ }^{26}$, International Study of Infarct Survival

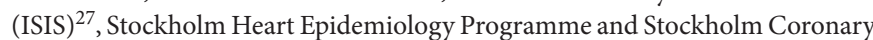
Artery Risk Factor study SHEEP/SCARF ${ }^{28,29}$, Precocious Coronary Artery Disease Transmission Disequilibrium Test (PROCARDIS TDT) cohort $^{30}$, Gruppo Italiano per lo Studio della Sopravvivenza nell'Infarto Miocardico Prevenzion (GISSI-P) ${ }^{31}$, Academic Medical Center Amsterdam Premature Atherosclerosis Study (AMC-PAS) ${ }^{32}$, The Hellenic study of Interactions between SNPs \& Eating in Atherosclerosis Susceptibility (THISEAS) ${ }^{33}$, and 3,359 cases and 2,828 controls of South Asian ancestry from the PROMIS ${ }^{24}$ and INTERHEART ${ }^{34}$ studies.

Selection of replication SNPs. SNPs were ranked by the association $P$ value in the discovery meta-analysis. The genotype clusters for all SNPs with an association with CAD of $P<1.0 \times 10^{-4}$ were manually checked, and 13 SNPs were removed. With the exception of rs9349379 at the PHACTR1 locus, SNPs that were located within a locus that had been previously reported to be associated with CAD (Table 1), or SNPs in strong LD $\left(r^{2}>0.5\right)$ with the most significant SNP at each locus, were removed. All remaining SNPs with $P<3.0 \times$ $10^{-5}$ in either the European studies only or South Asian studies or with $P<$ $1.0 \times 10^{-4}$ in the all-studies meta-analysis were considered for replication. When a SNP could not be multiplexed, alternative tagging SNPs $\left(r^{2}>0.8\right)$ were considered.

Genotyping in the replication studies. Genotyping of the replication samples was performed by primer extension and MALDI-TOF mass spectrometry using Sequenom iPLEX technology as two multiplexes containing 59 replication SNPs and three gender-specific polymorphisms. At the Centre National de Génotypage (CNG), the assay for rs9349379 was replaced by a Kaspar SNP assay supplied by KBioscience Ltd. The PROMIS replication genotypes included data from 1,005 Illumina Human660 arrays which were not available during the discovery GWAS. The COROGENE replication genotypes were obtained in silico from Illumina Human670 GWAS data.

The allelic intensities of each SNP assay were plotted and, where necessary, genotypes were manually called. SNPs with genotype calls that deviated from Hardy-Weinberg equilibrium with $P<8.5 \times 10^{-4}$ or had minor allele frequency $<1 \%$ were excluded. The call rate cutoff for SNPs was empirically established in each study. SNP genotypes that passed quality control were used to determine a study-specific sample rate cutoff. Details of replication genotyping and related quality control steps are summarized in Supplementary Table 2 . The PROCARDIS TDT study was a family based collection, and an additional quality control step was applied whereby families with greater than three Mendelian misinheritances were excluded.

Statistical analyses. Replication analyses. Logistic regression models were used to perform association analyses of CAD and to estimate the per-allele effect and standard error. The family based transmission disequilibrium test was used to estimate the association in the PROCARDIS TDT samples. This test is robust to the presence of population structure and was performed using TRANSMIT (see URLs) written by David Clayton. 
Replication meta-analysis. A fixed-effects inverse-variance-weighted meta-analysis was used to combine the results for each SNP across all replication studies with available data. Heterogeneity between all studies and between the European and the South Asian studies was assessed using Cochran's $Q$ statistic.

Conditional analysis. Conditional analyses were performed in each of the discovery studies. The position of the recombination hotspots flanking each of the SNPs selected for replication was determined, and those SNPs that were located between the two flanking hotspots were tested for association with CAD with the equivalent model used in the discovery analysis but conditioning on the genotype of the SNP chosen for replication.

eQTL analyses. Tissue samples for gene expression biobanks were obtained as described previously ${ }^{35}$. Briefly, tissue biopsies were taken from patients undergoing carotid endartectomy (plaque $n=117$ ) or valve surgery (liver $n=152$, aorta media $n=117$, aorta adventitia $n=103$ and mammary artery $n=88$ ). Extracted RNA was hybridized to Affymetrix HG-U133 plus 2.0 microarrays (plaque) or Affymetrix ST 1.0 Exon arrays (liver, aorta and mammary artery), and obtained scans were robust multichip average (RMA) normalized and $\log 2$ transformed. DNA was extracted from circulating blood cells and hybridized to Illumina Human610w-Quad Beadarrays. In the MuTHER study, RNA levels were measured in lymphoblastoid cell lines $(n=826)$, skin $(n=705)$ and fat biopsies $(n=825)$ from 850 well-phenotyped female twins (1/3 MZ and 2/3 DZ) from the TwinsUK resource using Illumina's wholegenome expression array HumanHT-12 version 3 as previously described ${ }^{36}$. Genotyping was performed in parallel using Illumina's whole-genome arrays. Associations between genotype and expression of genes were assessed using an additive linear model.

22. Broadbent, H.M. et al. Susceptibility to coronary artery disease and diabetes is encoded by distinct, tightly linked SNPs in the ANRIL locus on chromosome 9p. Hum. Mol. Genet. 17, 806-814 (2008).
23. Heart Protection Study Collaborative Group. MRC/BHF Heart Protection Study of cholesterol lowering with simvastatin in 20,536 high-risk individuals: a randomised placebo-controlled trial. Lancet 360, 7-22 (2002).

24. Saleheen, D. et al. The Pakistan Risk of Myocardial Infarction Study: a resource for the study of genetic, lifestyle and other determinants of myocardial infarction in South Asia. Eur. J. Epidemiol. 24, 329-338 (2009).

25. Chambers, J.C. et al. Common genetic variation near $M C 4 R$ is associated with waist circumference and insulin resistance. Nat. Genet. 40, 716-718 (2008).

26. Ripatti, S. et al. A multilocus genetic risk score for coronary heart disease case-control and prospective cohort analyses. Lancet 376, 1393-1400 (2010).

27. Clarke, R. et al. Lymphotoxin-alpha gene and risk of myocardial infarction in 6,928 cases and 2,712 controls in the ISIS case-control study. PLoS Genet. 2, e107 (2006).

28. Reuterwall, C. et al. Higher relative, but lower absolute risks of myocardial infarction in women than in men: analysis of some major risk factors in the SHEEP study. The SHEEP Study Group. J. Intern. Med. 246, 161-174 (1999).

29. Samnegard, A. et al. Serum matrix metalloproteinase- 3 concentration is influenced by MMP-3 -1612 5A/6A promoter genotype and associated with myocardial infarction. J. Intern. Med. 258, 411-419 (2005).

30. PROCARDIS Consortium. A trio family study showing association of the lymphotoxinalpha N26 (804A) allele with coronary artery disease. Eur. J. Hum. Genet. 12 770-774 (2004).

31. GISSI-Prevenzione Investigators. Dietary supplementation with n-3 polyunsaturated fatty acids and vitamin E after myocardial infarction: results of the GISSI-Prevenzione trial. Gruppo Italiano per lo Studio della Sopravvivenza nell'Infarto miocardico. Lancet 354, 447-455 (1999).

32. Trip, M.D. et al. Frequent mutation in the ABCC6 gene (R1141X) is associated with a strong increase in the prevalence of coronary artery disease. Circulation 106, 773-775 (2002).

33. Theodoraki, E.V. et al. Fibrinogen beta variants confer protection against coronary artery disease in a Greek case-control study. BMC Med. Genet. 11, 28 (2010).

34. Mente, A. et al. Metabolic syndrome and risk of acute myocardial infarction a case-control study of 26,903 subjects from 52 countries. J. Am. Coll. Cardiol. 55, 2390-2398 (2010).

35. Folkersen, L. et al. Association of genetic risk variants with expression of proximal genes identifies novel susceptibility genes for cardiovascular disease. Circ. Cardiovasc. Genet. 3, 365-373 (2010).

36. Stranger, B.E. et al. Population genomics of human gene expression. Nat. Genet. $\mathbf{3 9}$, 1217-1224 (2007) 